\title{
Instantons and Geometric Invariant Theory
}

\author{
S. K. Donaldson
}

All Souls College, Oxford, United Kingdom and The Institute for Advanced Study, Princeton, NJ 08540. USA

\begin{abstract}
We show that the Yang-Mills instantons can be described in terms of certain holomorphic bundles on the projective plane. The proof uses explicit matrix descriptions arising from monads and an analysis of the corresponding groups of symmetries.
\end{abstract}

In this note we show that the parameter spaces of instantons on $\mathbb{R}^{4}$ can be given a purely complex algebraic description. Precisely, let $G$ be one of the classical groups $\mathrm{SU}(\ell), \mathrm{SO}(\ell), \mathrm{Sp}(\ell)$, and $k \geqq 0$ be minus the Pontrayagin index (we work with anti self-dual connections) of a $G$ bundle $P$ over $S^{4}=\mathbb{R}^{4} \cup\{\infty\}$. Denote by $\tilde{M}(G, k)$ the moduli space whose points represent isomorphism classes of pairs:

(anti self-dual $G$-connection on $P$, isomorphism $P_{\infty} \cong G$ ).

These "framed" moduli spaces are in some respects more natural than the usual moduli of connections alone: $M(G, k)=\tilde{M}(G, k) / G$, and are manifolds of dimension $4 \ell k, 4(\ell-2) k, 4(\ell+1) k$ respectively, for $k$ sufficiently large.

Now fix a complex structure on $\mathbb{R}^{4}$ compatible with the metric and compactify in a different way to the complex projective plane:

$$
\mathbb{R}^{4} \cong \mathbb{C}^{2} \subseteq \mathbb{C} \mathbb{P}^{2}=\mathbb{C}^{2} \cup \ell_{\infty}
$$

Consider analogously the moduli space:

$$
\mathcal{O} \tilde{M}\left(G^{\mathbb{C}}, k\right)
$$

of holomorphic bundles on $\mathbb{C P}^{2}$ for the associated complex group, trivial on the line at $\infty, \ell_{\infty}$ and with a fixed holomorphic trivialization there. Then we shall prove:

Theorem. There is a natural one-to-one correspondence:

$$
\tilde{M}(G, k) \underset{R}{\stackrel{\cong}{\rightrightarrows}} \mathcal{O M}\left(G^{\mathbb{C}}, k\right) \text {. }
$$

(One consequence of this correspondence is that, interchanging the evident symmetry groups, we get an action of $\mathrm{SO}(4)$ on $\mathcal{O} \tilde{M}$ and $\operatorname{SL}_{2}(\mathbb{C})$ on $\tilde{M}$.) 
The map $R$ can be defined using the Penrose fibration:

$$
\mathbb{C} \mathbb{P}^{3} \stackrel{\pi}{\rightarrow} S^{4}
$$

Fixing a complex structure on $\mathbb{R}^{4}$ corresponds to fixing a complex plane $P$ in $\mathbb{C P}^{3}$ through the fiber $\ell_{\infty}=\pi^{-1}(\infty)$, the restriction of $\pi$ then gives the isomorphism $P \backslash \ell_{\infty} \rightarrow \mathbb{R}^{4}$. An anti self-dual connection on $S^{4}$ lifts, by the Ward correspondence, to a holomorphic bundle on $\mathbb{C P}^{3}$ and a trivialization at $\infty \in S^{4}$ lifts to a trivialization over $\ell_{\infty}$; then $R$ is defined by restricting this holomorphic bundle to the plane $P$. Taking this point of view Atiyah, in [1], noted that restriction gave an injection to an open subset of $\mathcal{O} \tilde{M}$.

The first reasons for believing that more should be true were analytical and independent of twistor methods. In [4] it was shown that the anti-self-dual connections on, say, $\mathbb{C P}^{2}$ with its standard metric correspond to certain holomorphic bundles; and it seems likely that similar techniques could be applied to prove our present theorem - regarding the flat metric on $\mathbb{C}^{2}$ as a metric on $\mathbb{C P}^{2}$ singular along $\ell_{\infty}$, and the trivialization there as giving boundary data for the differential equation discussed in [4]. However, we have not developed such an approach since it became apparent that one could give an elementary proof starting from the wellknown "ADHM construction." Indeed, after reviewing this construction (Sect. 1) and describing a general principle (Sect. 2), our proof is essentially a single observation.

This complex description of instantons is in some ways more manageable than the usual real algebraic statement of the ADHM classification. For example, we can see that (at least for groups $\mathrm{SU}(\ell)$ ) the moduli spaces of instantons are connected. This follows from the proofs by Barth and Hulek of the connectivity of the moduli spaces $M_{S}$ of stable bundles on $\mathbb{C P}^{2}([3,5,10,4.2])$. In fact, these proofs go by reducing first to the bundles trivial on a fixed line. There are dense Zariski open subsets:

$$
\begin{aligned}
& U \subseteq \mathcal{O} \tilde{M}, \\
& V \subseteq M_{S},
\end{aligned}
$$

representing stable bundles trivial on $\ell_{\infty}$, such that $U$ fibres over $V$ with connected fiber $\operatorname{SL}(\ell, \mathbb{C}) / \mathbb{Z}_{\ell}$, hence the connectivity of $M_{S}$ implies that of $\mathcal{O} \tilde{M}$. Taubes $[11]$ has recently proved this connectivity (for groups $\mathrm{SU}(2), \mathrm{SU}(3)$ ) by more direct methods. Conversely then Taubes' results give a new proof of the connectivity of certain moduli of stable bundles.

\section{Monads and the ADHM Construction}

Here we review some standard material, mostly in order to fix our notation. We work with connections for the group $G=\mathrm{SU}(\ell)$ and correspondingly holomorphic $\operatorname{SL}(\ell, \mathbb{C})$ bundles.

Bundles on $\mathbb{C P}^{2}$. Take homogeneous coordinates $[X]=(x ; y ; z)$ for the projective plane $\mathbb{C} \mathbb{P}^{2}$, with $z=0$ defining the line at $\infty$. Then by a monad we mean linear maps 
for each $X \in \mathbb{C}^{3}$ :

$$
H \stackrel{A_{X}}{\longrightarrow} K K \stackrel{B_{X}}{\longrightarrow} L
$$

between complex vector spaces of dimensions $k, 2 k+\ell, k$ respectively, depending linearly on $X$ :

$$
\begin{aligned}
& A_{X}=A_{x} x+A_{y} y+A_{z} z, \\
& B_{X}=B_{x} x+B_{y} y+B_{z} z,
\end{aligned}
$$

such that for each $X$ the composite $B_{X} A_{X}: H \rightarrow L$ is zero. This gives six equations for the maps $A_{x}, A_{y}, A_{z} ; B_{x}, B_{y}, B_{z}$. We say that the monad is non-degenerate it for each non-zero $X, A_{X}$ is injective and $B_{X}$ is surjective. In this case, the monad defines a holomorphic bundle on $\mathbb{C P}^{2}$ whose fiber at $[X]$ is the vector space $\operatorname{Ker} B_{X} / \operatorname{Im} A_{X}$. It is known that any bundle on $\mathbb{C P}^{2}$ trivial on some line comes from such a monad, unique up to the action of $\mathrm{GL}(H) \times \mathrm{GL}(K) \times \mathrm{GL}(L)$ (cf. [10, p. 249 and Lemma $4.1 .3 ; 3,5])$.

Now consider bundles trivial on the fixed line $z=0$. The condition on the monad for this is that the composite:

$$
B_{x} A_{y}=-B_{y} A_{x} \text { is an isomorphism ([10, Lemma 4.2.3]). }
$$

For such bundles we can reduce the symmetry of the monad by choosing bases for $H$, $K, L$ so that $B_{x} A_{y}=1_{k}=$ identity matrix. Further, the three conditions:

$$
B_{x} A_{y}=-B_{y} A_{x}, \quad B_{y} A_{y}=0, \quad B_{x} A_{x}=0
$$

imply that we can normalize the bases so that the maps are given by:

$$
\begin{aligned}
A_{x}= & \left(\begin{array}{l}
1 \\
0 \\
0
\end{array}\right) \begin{array}{l}
\downarrow k \\
\uparrow \ell
\end{array} \quad A_{y}=\left(\begin{array}{l}
0 \\
1 \\
0
\end{array}\right) \\
& \stackrel{\leftrightarrow}{k} \\
B_{x}= & \left(\begin{array}{lll}
0 & 1 & 0
\end{array}\right) \quad B_{y}=\left(\begin{array}{lll}
-1 & 0 & 0
\end{array}\right)
\end{aligned}
$$

The two equations $B_{z} A_{y}=-B_{y} A_{z}, B_{z} A_{x}=-B_{x} A_{z}$ mean that we have:

$$
A_{z}=\left(\begin{array}{c}
\alpha_{1} \\
\alpha_{2} \\
a
\end{array}\right), \quad B_{z}=\left(-\alpha_{2} \alpha_{1} b\right), \quad \text { say, }
$$

and we are left with the remaining matrix equation:

$$
B_{z} A_{z}=0 \Leftrightarrow\left[\alpha_{1}, \alpha_{2}\right]+b a=0 .
$$

The restriction of the bundle to the line $z=0$ has a natural frame given by the last $\ell$ basis vectors of $K \cong \mathbb{C}^{2 k+\ell}$, and so we have the description of Barth:

Proposition 1. The space $\mathcal{O} \tilde{M}(\mathrm{SL}(\ell, \mathbb{C}), k)$ is the quotient of the set of matrices 
$\left(\alpha_{1}, \alpha_{2}, a, b\right)$ satisfying:

(i) $\left[\alpha_{1}, \alpha_{2}\right]+b a=0$

(ii) for all $\lambda, \mu \in \mathbb{C}^{2},\left(\begin{array}{c}\alpha_{1}+\lambda \\ \alpha_{2}+\mu \\ a\end{array}\right)$ is injective and $\left(\lambda-\alpha_{1} \alpha_{2}-\mu b\right)$ is surjective, by the natural action of $\operatorname{GL}(k, \mathbb{C})$ :

$$
\left.\begin{array}{rl}
\alpha_{i} \mapsto p \alpha_{i} p^{-1} \\
a \mapsto a p^{-1} \\
b \mapsto p b
\end{array}\right\} p \in \mathrm{GL}(k, \mathbb{C})
$$

Bundles on $\mathbb{C P}^{2}$ and the ADHM Construction (See $[1,2]$ for more details.)

Now regard $\mathbb{C P} \mathbb{P}^{2}$ as a plane in $\mathbb{C P}^{3}$ by taking a fourth coordinate $w$. We consider analogously monads for bundles on $\mathbb{C P}^{3}$ trivialized on the line $\ell_{\infty}=(z=w=0)$, so we set:

$$
A_{X}=A_{x} x+A_{y} y+A_{z} z+A_{w} w, \quad \text { etc. }
$$

having matrix representations:

$$
\begin{aligned}
& A_{x}=\left(\begin{array}{l}
1 \\
0 \\
0
\end{array}\right), \quad A_{y}=\left(\begin{array}{l}
0 \\
1 \\
0
\end{array}\right), \quad A_{z}=\left(\begin{array}{l}
\alpha_{1} \\
\alpha_{2} \\
a
\end{array}\right), \quad A_{w}=\left(\begin{array}{c}
\hat{\alpha}_{1} \\
\hat{\alpha}_{2} \\
\hat{a}
\end{array}\right), \\
& B_{x}=\left(\begin{array}{lll}
0 & 1 & 0
\end{array}\right), \quad B_{y}=\left(\begin{array}{lll}
-1 & 0
\end{array}\right), \quad B_{z}=\left(-\alpha_{2} \alpha_{2} b\right), \quad B_{w}=\left(\begin{array}{lll}
-\hat{\alpha}_{2} & \hat{\alpha}_{1} b
\end{array}\right)
\end{aligned}
$$

with non-degeneracy conditions and three equations:

(a) $\left[\alpha_{1}, \alpha_{2}\right]+b a=0$,

(b) $\left[\hat{\alpha}_{1}, \hat{\alpha}_{2}\right]+\hat{b} \hat{a}=0$,

(c) $\left[\alpha_{1}, \hat{\alpha}_{2}\right]+\left[\hat{\alpha}_{1}, \alpha_{2}\right]+b \hat{a}+\hat{b} a=0$.

Finally, we introduce the "real" bundles corresponding to instantons. The quaternions: $\mathbb{R}(1, I, J, K=I J)$ act on $\mathbb{C}^{4}$,

$$
\begin{aligned}
& I(x, y, z, w)=(i x, i y, i z, i w), \\
& J(x, y, z, w)=(\bar{y},-\bar{x}, \bar{w},-\bar{z}),
\end{aligned}
$$

and we seek monads for bundles on $\mathbb{C P}^{3}$ with an isomorphism $\overline{J^{*}(\mathscr{E})} \cong \mathscr{E} *$, trivial on all real lines. Then the space $K$ acquires a Hermitian metric and $L$ becomes isomorphic to $\bar{H}^{*}$, so we may consider the adjoint $A^{*}$ as mapping $K$ to $L$ and, for $X \in \mathbb{C}^{4}$, the complex linear map:

$$
\mathscr{A}_{X}=A_{X}^{*} \oplus B_{X}: K \rightarrow L \oplus L .
$$


The quaternions act on $L \oplus L$ by:

$$
I\left(\begin{array}{l}
\ell \\
m
\end{array}\right)=\left(\begin{array}{c}
-i \ell \\
i m
\end{array}\right), \quad J\left(\begin{array}{l}
\ell \\
m
\end{array}\right)=\left(\begin{array}{c}
-m \\
\ell
\end{array}\right),
$$

and we demand that $\mathscr{A}$ commutes with the quaternions, that is:

$$
\mathscr{A}_{q X}(v)=q \mathscr{A}_{X}(v) \text { for } v \in K, q \in \mathbb{H} \text {. }
$$

This implies that the kernel of $\mathscr{A}_{X}$ is constant along the fibers of the Penrose map:

$$
\pi: \mathbb{C} \mathbb{P}^{3} \rightarrow H \mathbb{P}^{1} \cong S^{4},
$$

and descends to give the fiber of the bundle over $S^{4}$ carrying an anti-self-dual connection.

In our matrix description we suppose the bases are chosen in the obvious way, compatible with the real structures (so in particular the induced frame at $\infty$ is orthonormal). Then the reality condition becomes:

$$
\left(\begin{array}{c}
-B_{z} z-B_{w} w \\
A_{z}^{*} \bar{z}+A_{w}^{*} \bar{w}
\end{array}\right)=J\left(\begin{array}{c}
A_{z}^{*} \bar{z}+A_{w}^{*} \bar{w} \\
B_{z} z+B_{w} w
\end{array}\right)=\left(\begin{array}{c}
A_{z}^{*} w-A_{w}^{*} z \\
B_{z} \bar{w}-B_{w} \bar{z}
\end{array}\right),
$$

that is, $A_{w}^{*}=B_{z}, A_{z}^{*}=-B_{w}$.

So we can eliminate the , variables:

$$
\hat{\alpha}_{1}=-\alpha_{2}^{*}, \quad \hat{\alpha}_{2}=\alpha_{1}^{*} ; \quad \hat{a}=b^{*}, \quad \hat{b}=-a^{*} .
$$

Moreover, the non-degeneracy conditions for a monad of this type on $\mathbb{C P}^{3}$ are the same as those ((ii) of Proposition 1) for the restriction to $\mathbb{C P} \mathbb{P}^{2}$; since $\mathscr{A}_{X}$ is surjective if and only if $\mathscr{A}_{q X}=q \mathscr{A}_{X}$ is (for $q \in \mathbb{H}^{*}$ ), and $\mathbb{C P}^{2}$ sweeps out $\mathbb{C P}^{3}$ under $\mathbb{H}^{*}$. Substituting into the three equations (a), (b), (c); (a) and (b) become adjoints and (c) becomes:

$$
\left[\alpha_{1}, \alpha_{1}^{*}\right]+\left[\alpha_{2}, \alpha_{2}^{*}\right]+b b^{*}-a^{*} a=0 .
$$

The whole setup is invariant under $U(k)$ and we are able to state the description of Atiyah, Drinfeld, Hitchin and Manin:

Proposition 2. The space $\tilde{M}(\mathrm{SU}(\ell), k)$ is the quotient of the set of complex matrices $\left(\alpha_{1}, \alpha_{2}, a, b\right)$, satisfying conditions (i), (ii) of Proposition 1, and also :

(iii) $\left[\alpha_{1}, \alpha_{1}^{*}\right]+\left[\alpha_{2}, \alpha_{2}^{*}\right]+b b^{*}-a^{*} a=0$

by the action of $U(k)$

\section{Geometric Invariant Theory and Closest Points}

Next we describe some general machinery which will lead immediately to the proof of our theorem. We give a minimal account and refer to two articles [7, 9] which contain extensive discussions and applications of these ideas. The precise fact that we use is due to Kempf and Ness [6].

Suppose the group $\operatorname{GL}(k, \mathbb{C})$ acts by some linear representation on $\mathbb{C}^{N}$ with the 
subgroup $\mathrm{U}(k)$ acting isometrically. Then in Mumford's Geometric Invariant theory there is a definition of a "stable point" ("property stable" in [8]) in $\mathbb{C}^{N}$. Roughly speaking, the $\mathrm{GL}(k)$ quotient of the set of stable points has a good structure. The definition is this:

Definition. The point $x$ in $\mathbb{C}^{N}$ is stable for $\operatorname{GL}(k, \mathbb{C})$ if and only if the map $\mathrm{GL}(k) \rightarrow \mathbb{C}^{N} ; g \mapsto g x$ is proper.

In particular, the $\mathrm{GL}(k)$ orbit of a stable point $x$ is closed in $\mathbb{C}^{N}$ and so contains a vector, say $x$ itself, closest to the origin. Writing down the infinitesimal form of this gives an equation satisfied by $x: \mu(x)=0$, where $\mu: \mathbb{C}^{N} \rightarrow \mathfrak{U}(k)$. For example, take the adjoint representation of $\mathrm{GL}(k)$. Then if a matrix $\alpha$ is closest to the origin in its orbit $\left\{g \propto g^{-1}\right\}$ :

$$
\begin{aligned}
\delta\left\|g \alpha g^{-1}\right\|^{2} & =2\left\langle\delta\left(g \alpha g^{-1}\right), \alpha\right\rangle=2\langle[\delta g, \alpha], \alpha\rangle \\
& =2\left\langle\delta g,\left[\alpha, \alpha^{*}\right]\right\rangle=0 \text { for all } \delta g,
\end{aligned}
$$

that is: $\mu(\alpha) \equiv\left[\alpha, \alpha^{*}\right]=0$.

Moreover, it can be shown [6] that this closest point is unique in the GL(k) orbit up to the action of the unitary group $\mathrm{U}(k)$, as the reader can check in the example above. So we have the following general principle of Kempf and Ness.

Proposition 3. For any representation as above and any GL(k) invariant subset $W \subset \mathbb{C}^{N}$, all of whose points are stable for the action, the quotient $W / \mathrm{GL}(k)$ is naturally equivalent to the quotient $\left(\mu^{-1}(0) \cap W\right) / \mathrm{U}(K)$.

In practice the stability of a point can be tested by the:

Hilbert Criterion ([8, Theorem 2.1, Proposition 2.2]). The point $x$ is stable for $\mathrm{GL}(k, \mathbb{C})$ if and only if it is so for all 1-parameter subgroups. That is, iffor all $g \in \mathrm{GL}(k)$ and integers $\left\{w_{i}\right\}_{i=1}^{k}$ (not all zero):

$$
g \operatorname{diag}\left(t^{w_{1}}, \ldots, t^{w_{k}}\right) g^{-1} \cdot x \rightarrow \infty \quad \text { as } t \rightarrow \infty .
$$

Now we apply these ideas to our problem. Take $\operatorname{GL}(k, \mathbb{C})$ acting on the vector space of matrices $\left(\alpha_{1}, \alpha_{2}, a, b\right)$ as in Proposition 1 . The unitary group preserves the obvious norm and computing the quantity $\mu\left(\alpha_{1}, \alpha_{2}, a, b\right)$ as before, we get fortuitously:

$$
\begin{aligned}
\delta\left\|\left(\alpha_{1}, \alpha_{2}, a, b\right)\right\|^{2} & =\delta\left\|\alpha_{1}\right\|^{2}+\delta\left\|\alpha_{2}\right\|^{2}+\delta\|a\|^{2}+\delta\|b\|^{2} \\
& =2\left\langle\delta g,\left[\alpha_{1}, \alpha_{1}^{*}\right]+\left[\alpha_{2}, \alpha_{2}^{*}\right]+b b^{*}-a^{*} a\right\rangle,
\end{aligned}
$$

i.e.,

$$
\mu\left(\alpha_{1}, \alpha_{2}, a, b\right)=\left[\alpha_{1}, \alpha_{1}^{*}\right]+\left[\alpha_{2}, \alpha_{2}^{*}\right]+b b^{*}-a^{*} a .
$$

Moreover, we have:

Lemma. Suppose the matrices $\left(\alpha_{1}, \alpha_{2}, a, b\right)$ correspond to an element of $\mathcal{O} \tilde{M}(\mathrm{SL}(\ell, \mathbb{C}), k)$; that is, satisfy the two conditions (i), (ii) of Proposition 1. Then the point $\left(\alpha_{1}, \alpha_{2}, a, b\right)$ is stable for the action of $\mathrm{GL}(k, \mathbb{C})$. 
Proof. Suppose, on the contrary, that some $\left(\alpha_{1}, \alpha_{2}, a, b\right)$ is not stable. Then by the Hilbert criterion and the $\mathrm{GL}(k)$ invariance we may suppose that:

$$
p_{t}\left(\alpha_{1}, \alpha_{2}, a, b\right)=\left(p_{t} \alpha_{1} p_{t}^{-1}, p_{t} \alpha_{2} p_{t}^{-1}, a p_{t}^{-1}, p_{t} b\right)
$$

is bounded as $t \rightarrow \infty$, for $p_{t}=\operatorname{diag}\left(t^{w_{1}} t^{w_{2}} \ldots t^{w_{k}}\right) w_{1} \geqq w_{2} \geqq \cdots \geqq w_{k}$. Suppose first that some $w_{i}$ is positive, say $w_{1}>0$ and $w_{1}=w_{2}=\cdots=w_{s}>w_{s+1}$. Then considering the action on $b$ we must have the first $s$ rows identically zero;

$$
b=\left(\begin{array}{l}
0 \\
*
\end{array}\right)^{\uparrow s}
$$

hence

$$
b a=\left(\begin{array}{l}
0 \\
*
\end{array}\right)
$$

Likewise $p$ acts by $t^{w_{1}-w_{J}}$ on the $(i, j)^{\text {th }}$-entry of the $\alpha$ 's so:

$$
\alpha_{i}=\left(\begin{array}{cc}
M_{i} & 0 \\
* & *
\end{array}\right) \text { say, and }\left[\alpha_{1}, \alpha_{2}\right]=\left(\begin{array}{cc}
{\left[M_{1}, M_{2}\right]} & 0 \\
* & *
\end{array}\right)
$$

Suppose now $\left(\alpha_{1}, \alpha_{2}, a, b\right)$ satisfy condition (i): $\left[\alpha_{1}, \alpha_{2}\right]+b a=0$. Then $M_{1}, M_{2}$, and so $M_{1}^{T}, M_{2}^{T}$ commute-hence have a common eigenvector $v ;\left(\lambda-M_{1}^{T}\right) v=$ $\left(M_{2}^{T}-\mu\right) v=0$. Then the row vector $\left(v^{T} 0\right)$ annihilates the image of $\left(\lambda-\alpha_{1} \alpha_{2}-\mu b\right)$, contradicting property (ii).

In precisely the same way if some $w_{i}$ is negative, we get a vector in the kernel of $\left(\begin{array}{c}\alpha_{1}+\lambda \\ \alpha_{2}+\mu \\ a\end{array}\right)$. Hence every point satisfying (i), (ii) is stable. (In fact, for points in the closed variety satisfying (i), stability is equivalent to (ii).)

With this small lemma we have the proof of the theorem, for $G=\operatorname{SU}(\ell)$, immediately in our hands. For we take $W$ to be the set of matrices $\left(\alpha_{1}, \alpha_{2}, a, b\right)$ satisfying the conditions (i), (ii) and substitute Propositions 1 and 2 into Proposition 3 , exploiting the fact that the equation $\mu\left(\alpha_{1}, \alpha_{2}, a, b\right)=0$ is the extra condition (iii).

For the sympletic and orthogonal groups, one can follow the same path, working with the corresponding self-dual monads. Alternatively, argue in the obvious way, regarding a symplectic or orthogonal holomorphic bundle as a bundle $\mathscr{E}$ with an isomorphism $\stackrel{f}{\mathscr{f}} \rightarrow \mathscr{E} *$. For each trivialization on $\ell_{\infty} \mathscr{E}, \mathscr{E} *$ both carry unitary antiself-dual connections, and if the trivializations are consistent with $f$, the connections will, by the uniqueness, be also - that is, the connection then reduces to the corresponding compact group.

\section{3}

We have given this proof in an unashamedly computational, coordinate based, fashion. However, it is interesting to note that the discussion of Sect. 2 can be abstracted and the map $\mu$ then appears as a moment map for the action of $\mathrm{U}(k)([7$, 9]). On the other hand, as explained in [4], the anti self-duality equations in the 
context of holomorphic bundles can also be interpreted as the vanishing of a moment map, but this time for the infinite dimensional gauge group. Perhaps the geometrical reason for this coincidence lies in the ideas of E. Corrigan and W. Nahm on the dual nature of the ADHM construction--transforming commutator equations for differential operators into formally similar commutator equations for matrices.

\section{References}

1. Atiyah, M. F.: The geometry of Yang Mills fields. In: Fermi Lectures. Scuola Normale Pisa 1979

2. Atiyah, M. F., Drinfeld, V., Hitchin, N. J., Manin, Yu. I. : Construction of instantons. Phys. Lett. 65A, 185 (1978)

3. Barth, W.: Moduli of vector bundles on the projective plane. Invent. Math. 42, 63-91 (1977)

4. Donaldson, S. K. : Anti self-dual Yang-Mills connections on complex algebraic surfaces and stable vector bundles. Proc. Lond. Math. Soc. (submitted)

5. Hulek, K. : On the classification of stable rank-r vector bundles on the projective plane. In: Proc. Nice conference on vector bundles and differential equations, Hirschowitz, A. (ed.). Boston: Birkhäuser 113-142 (1983)

6. Kempf G., Ness, L. : Lengths of vectors in representation spaces. In: Lecture Notes in Mathematics, Vol. 732, Berlin, Heidelberg, New York: Springer 233-244 (1978)

7. Kirwan, F. C.: The cohomology of quotients in symplectic and algebraic geometry. Oxford University, preprint

8. Mumford, D. : Geometric invariant theory. Erg. Math. 34, Berlin, Heidelberg, New York: Springer 1965

9. Ness, L. : A stratification of the null cone by the moment map. Am. J. Math. (to appear)

10. Okonek, C., Schneider, M., Spindler, H. : Vector bundles on complex projective spaces. Boston: Birkhäuser 1980

11. Taubes, C. H. : Path connected Yang-Mills moduli spaces. U.C. Berkeley preprint

Communicated by A. Jaffe

Received October 21, 1983; in revised form November 17, 1983 